\title{
Perihematomal glutamate level is associated with the blood-brain barrier disruption in a rabbit model of intracerebral hemorrhage
}

\author{
Guofeng $\mathrm{Wu}^{1^{*+}}$, Shujie Sun ${ }^{2+}$, Fei Sheng ${ }^{1}$, Likun Wang ${ }^{1}$ and Fan Wang ${ }^{1}$
}

\begin{abstract}
Objective: To observe the relationship between the perihematomal glutamate levels and the blood-brain barrier (BBB) permeability in a rabbit model of intracerebral hemorrhage (ICH).

Methods: Seventy-two rabbits were randomly divided into an intracerebral hemorrhage (ICH) model group and a normal control (NC) group, and each group of 36 rabbits was subsequently divided into 6, 12, 18, 24, 48 and $72 \mathrm{~h}$ groups ( $n=6$ each). An ICH model was induced by stereotactic injection of autologous, arterial, non-anticoagulated blood into rabbit basal ganglia. The same procedures were performed in the NC group, but blood was not injected. The rabbits were sacrificed at specific time points after the experiment began depending on their group. Perihematomal brain tissues were collected to determine glutamate levels, BBB permeability and brain water content (BWC).

Results: All of the assessed parameters were increased 6 hour after blood infusion and continued to gradually increase, peaking at 48 hours. Differences were observed when ICH values were compared with those of the NC group $(p<0.05)$.

Conclusions: Perihematomal glutamate increased significantly after ICH. High levels of glutamate are closely associated with BBB disruption and the brain edema. Therefore, glutamate may play an important role in the pathogenesis of secondary brain injury after (ICH).
\end{abstract}

Keywords: Intracerebral hemorrhage; Perihematomal glutamate; Brain water content; Blood-brain-barrier; Evans blue

\section{Introduction}

Spontaneous intracerebral hemorrhage $(\mathrm{ICH})$ is a devastating neurological disorder with considerable mortality and morbidity that accounts for $20 \%-30 \%$ of acute cerebral vascular disease. However, no currently available intervention has been shown to alter the outcome of patients who have suffered acute ICH (Parker et al. 2010). Secondary brain damage, which often occurs in the days following the initial hemorrhage, is closely associated with significant neurological deterioration (Miller et al. 2007). ICH negatively affects BBB permeability, which

\footnotetext{
*Correspondence: wuguofeng3013@sina.com

${ }^{\dagger}$ Equal contributors

'Department of Neurology, Affiliated Hospital, Guiyang Medical College, No. 28, Guiyijie Road, Liuguangmen, Guiyang City, Guizhou Province 550004, P R China

Full list of author information is available at the end of the article
}

results in neuronal dysfunction (Shi et al. 2011). A hallmark of ICH-induced brain injury, BBB disruption is accompanied by brain edema in the surrounding areas of $\mathrm{ICH}$. The degree of BBB breakdown has been directly correlated with late functional outcome (Lampl et al. 2005). A range of factors including inflammatory mediators, thrombin and hemoglobin breakdown products are involved in BBB disruption (Keep et al. 2008).

Recently, some studies have observed a relationship between the perihematomal glutamate levels and the secondary brain injury in $\mathrm{ICH}$ models, and the impact of glutamate on $\mathrm{ICH}$ patient outcome (Miller et al. 2007; Chiang et al. 2006; Hartings et al. 2008; Qureshi et al. 2003; Wang et al. 2008a). Glutamate accumulates during the early period of experimental hematoma, and the activation of N-methyl-D-aspartate (NMDA) receptors by 
glutamate can result in an influx of calcium $\left(\mathrm{Ca}^{2+}\right)$ and subsequent neuronal death (Qureshi et al. 2003; Lee et al. 2006). In cases of $\mathrm{ICH}$, increased levels of glutamate and aspartate that correlate with neurological status have been detected after subarachnoid hemorrhage (SAH) (Germano et al. 2007), and glutamate-mediated excitotoxicity is a major consequence of stroke (Hazell 2007). Blockade of NMDA or AMPA receptors could attenuate BBB disruption in focal cerebral ischemia, and treatment with magnesium, MK-801 and a combination of magnesium and MK-801 can reduce brain edema formation and can help restore BBB integrity after experimental diffuse brain injury (Imer et al. 2009). Reducing perihematomal glutamate level by minimally invasive procedures for intracerebral hematoma also decreases BBB permeability and BWC (Wu et al. 2011). These results demonstrate that secondary brain damage is associated with glutamate-related excitotoxicity, and glutamate levels are closely associated with $\mathrm{ICH}$ patient outcome. However, the exact relationships among elevated glutamate levels in perihematomal brain tissues, BBB permeability and brain edema have not been thoroughly investigated.

The objective of the current study was to determine the relationship between the perifocal glutamate levels and the BBB permeability in a rabbit model of $\mathrm{ICH}$.

\section{Methods and materials Materials \\ Main reagents}

Formamide (molecular formula: $\mathrm{HCONH}_{2}$, Chongqing Chuanjiang Chemical Reagent Factory, Chongqing, China), urethane (molecular formula: $\mathrm{C} 3 \mathrm{H} 7 \mathrm{NO} 2$, Wuxi Yangshan Biochemical), Evans blue (Beijing Hengye Zhongyuan Chemical), 4\% paraformaldehyde (Wuhan Boster Biological Technology), urokinase (Guangdong Livzon Pharmaceutical), glutamate (Sigma), derivatization reagent borate buffer (Agilent Technologies, USA), FMOC reagent Agilent PN5061-3337 (Agilent Technologies, USA), OPA reagent Agilent PN5061-3335 (Agilent Technologies, USA, 2,4-DNFB (Japan) and HPLC-grade acetonitrile and methanol (Germany) were used in this study.

\section{Main instruments}

We used the following instruments: a ZH-Lanxing BType rabbit stereotaxic Apparatus (Huaibei Zhenghua Biological Instrument \& Equipment), electronic scales (Satourious, Germany), a Rainbow Type-722 grating spectrophotometer (Shandong Gaomi Rainbow Analytical Instrument), a 5415R high-speed centrifuge (Frozen, Heraeus Company), micropipettes (Eppendorf), a 202-2 constant temperature oven (Shanghai Luda Laboratory Apparatus), a digital display thermostat water bath ( $\mathrm{HH}-2$;
Guohua Electric Appliance), a tabletop general centrifuge (TGL-16B; Shanghai Anting Scientific Instrument Factory), a $-80^{\circ} \mathrm{C}$ freezer (Forman Scientific Company), a refrigerator (Qingdao), a CT provided by the Guiyang Medical College, a high-performance liquid chromatograph (HP-1100; Agilent Technologies, USA), a G1315 A diode-array detector (DAD, Agilent Technologies, USA), a pH meter (410 A, ORION, USA), an Agilent 1313A Automatic Sampler (Agilent Technologies, USA), a column oven (Agilent Technologies, USA) and scales (Beijing Gangdong Hengye Instrument).

\section{Experimental groups}

The present study was approved by the Animal Care and Use Committee of Guiyang Medical College.

Seventy-two male rabbits $(2.8-3.4 \mathrm{~kg})$ were provided by the Animal Center of Guiyang Medical College. The animals were divided randomly into an $\mathrm{ICH}$ group and a $\mathrm{NC}$ group $(\mathrm{n}=36$ each), and they were equally divided into 6 subgroups $(\mathrm{n}=6$ each) that were sacrificed at 6 , $12,18,24,48$ or $72 \mathrm{~h}$ after ICH induction. ICH was induced in all animals in the model group.

\section{Animal preparation \\ ICH model preparation}

Rabbits were fasted for 12 hours and water restricted for 4 hours prior to the experiment. The rabbits were then anesthetized by injecting $20 \%$ urethane $(5 \mathrm{ml} / \mathrm{kg}$ ) into the ear vein. Slow breathing, a slow corneal reflex and no pain reaction were used as indicators of complete anesthetization. The head of the rabbit was then shaved to expose the skin for surgery.

The anesthetized rabbit was fastened to the stereotaxic apparatus, and the skin in the operation field was disinfected with $75 \%$ alcohol. A $3-\mathrm{cm}$ incision was made along the mid-line between the two post-orbital margins, and the subcutaneous fascia was stripped to expose the skull. A $3 \% \mathrm{H}_{2} \mathrm{O}_{2}$ solution was used to open the periosteum and expose the bregma and lambdoid sutures. The head was then adjusted to make the bregma $1.5 \mathrm{~mm}$ higher than the lambdoid suture. The position of the internal capsule was located according to the rabbit stereotaxic atlas. The coronal plane crossing the center of bregma was used as the coronal zero plane (AP0); A1 represented the coronal plane $1 \mathrm{~mm}$ rostral to the AP0, and the internal capsule was estimated to be between A5 and P2. The present experiment used the A1 level and the bregma as base points, with the puncture point $6 \mathrm{~mm}$ left of the coronal suture and $1 \mathrm{~mm}$ parallel to the sagittal suture. A hole was drilled in the skull, and a \#12 needle and a 1-ml syringe were used to deliver $0.8 \mathrm{ml}$ autologous arterial blood taken from the central ear artery. The syringe was then connected to a \#7 needle in without a tip. Air was completely removed from 
the syringe, leaving $0.3 \mathrm{ml}$ of blood. The \#7 needle was quickly inserted vertically $12 \mathrm{~mm}$ into the skull, and the blood was slowly injected into the basal ganglia. The injection lasted for approximately 3 minutes. The needle was left in place for $8 \mathrm{~min}$ after the injection to prevent blood backflow, and then the needle was slowly removed. Local hemostasis was induced by compression for $2 \mathrm{~min}$.

The drill hole was then covered using gutta-percha. A CT scan was performed 3 hours later. A hyperdensity shadow in the basal ganglia region without a shadow in the lateral ventricle was considered as a successful model of ICH.

After successful ICH induction confirmed by CT scan, the animals were returned to the housing facility. All the animals recovered from anesthesia within 5 hours after intravenous injection of $20 \%$ urethane. Exclusion criteria included visualization of back flow along the needle track, blood in the ventricle, and death.

\section{NC group treatment}

Procedures performed in the $\mathrm{ICH}$ group were identical in the NC group, but autologous arterial blood was not injected into the basal ganglia to induce intracerebral hematoma.

\section{Medical treatment of the animals}

Animals received an intramuscular injection of penicillin $(400,000 \mathrm{U})$ to prevent infection. They were housed as usual until they were sacrificed. No other medical treatment was administered.

\section{Intracerebral hematoma volume and neurological deficit score}

To demonstrate that ICH was successfully modeled, hematoma volume was measured with a CT scan, and a neurological deficit scale was used to assess neurological functions (Purdy et al. 1989). The scale included tests of motor function (Parker et al. 2010; Miller et al. 2007; Shi et al. 2011; Lampl et al. 2005), consciousness (Parker et al. 2010; Miller et al. 2007; Shi et al. 2011; Lampl et al. 2005), head turning (0-1), circling (0-1) and hemianopsia (0-1). A total score of 11 indicates maximum impairment (comatose or dead rabbit), whereas 2 denotes complete normality. Tests were conducted by an observer blinded to the animals. The tests were conducted by an observer blinded to the animal's group.

\section{Brain tissues preparation}

A $2 \%$ Evans blue $(2 \mathrm{ml} / \mathrm{kg})$ solution was injected into the ear vein 2 hours before euthanization. The animals were then anesthetized using $20 \%$ urethane, and the chest was quickly opened to expose the heart. A tube was inserted from the left ventricle into the aortic root, with a small hole cut in the right ventricle to allow the tube to exit. Rabbits were transcardially perfused with $400 \mathrm{ml} 0.9 \%$ sodium chloride solution until the fluid flowed clear, at which time they were perfused with $100 \mathrm{ml} 4 \%$ paraformaldehyde. The brain was then extracted and placed on ice. The needle track was used as the center to prepare coronal and sagittal sections, then the brain on the hematoma side was cut and divided into four parts: front-inner, front-outside, back-inner and back-outside. A total of $5 \mathrm{~mm}$ of brain tissue surrounding the hematoma was collected from each area. The front-inner and front-outside parts were used for amino acid testing, the back-inner part was used for assessing Evans blue content, and the back-outside part was used for testing BWC.

\section{Perihematomal glutamate level measurement}

Perihematomal glutamate content was determined using high-performance liquid chromatography.

\section{Chromatographic conditions}

A ZORBAX Eclipse-AAA $(4.6 \times 150 \mathrm{~mm}, 5 \mu \mathrm{m})$ chromatographic column was used. Mobile phase A was $40 \mathrm{mM}$ $\mathrm{Na}_{2} \mathrm{HPO}_{4}, \mathrm{pH} 7.8\left(5.5 \mathrm{~g} \mathrm{Na}_{2} \mathrm{HPO}_{4} \cdot \mathrm{H}_{2} \mathrm{O}+1 \mathrm{l}\right.$ water, $\mathrm{NaOH}$ was added to make the $\mathrm{pH} 7.8$ ) and mobile phase B was 45:45:10 (V/V/V) ACN: MeOH:water. The column was run with a flow rate of $2 \mathrm{ml} / \mathrm{min}$. Phase B increased from 0 to $57 \%$ between 0 and $18 \mathrm{~min}$ and from 57 to $100 \%$ between 18.1 and $18.6 \mathrm{~min}$. It remained at $100 \%$ between 18.6 and $22.3 \mathrm{~min}$ and decreased from $100 \%$ to $0 \%$ between $22.3 \mathrm{~min}$ and $23.2 \mathrm{~min}$. Between 23.2 and $26 \mathrm{~min}$, Phase B remained at $0 \%$. The column temperature was $40^{\circ} \mathrm{C}$, and the sampling volume was $10 \mu$ l. The diode array wavelength was $262 \mathrm{~nm}$, and the reference wavelength was $324 \mathrm{~nm}$.

\section{Derivative solution preparation}

A total of $25 \mathrm{mg}$ OPA was dissolved in $1 \mathrm{ml}$ methanol. Sodium borohydride buffer $(4 \mathrm{~mol} / \mathrm{l})$ was then added $(\mathrm{pH}$ 10.4), and the solution was stirred. The final solution was stored at $4^{\circ} \mathrm{C}$.

\section{Standard solution preparation}

Glutamate and $0.2 \mathrm{~mol} / \mathrm{l} \mathrm{NaHCO}_{3}(\mathrm{pH} 9.8)$ was used to make a 1 -g/l standard stock solution.

\section{Biosample preparation}

The brain tissue was defrosted, weighed and placed in a dry glass homogenizer. Dilute hydrochloric acid $(1: 5 \mathrm{w} / \mathrm{v}$, $0.1 \mathrm{mmol} / \mathrm{l})$ was added, and the homogenized brains were placed into an ultrasonicator (Temp: $4^{\circ} \mathrm{C}$; pulse for $2 \mathrm{~s}$, rest for $2 \mathrm{~s}$; intensity: 20\%; 15 times in total). Samples were then centrifuged at 1,200 rpm for $20 \mathrm{~min}$ at $4^{\circ} \mathrm{C}$. Borate saline buffer $(2.5 \mu \mathrm{l})$ was then added to 
the supernatant solution and mixed for $20 \mathrm{~min}$, followed by the addition of $0.5 \mu \mathrm{lOPA}$. The sample was then mixed for $30 \mathrm{~s}$ before FMOC $(0.5 \mu \mathrm{l})$ was added and the solution was mixed for another $30 \mathrm{~s}$. Finally, water $(32 \mu \mathrm{l})$ was added, and the final sample was mixed for $30 \mathrm{~s}$, and $10 \mu \mathrm{l}$ of the sample was used.

\section{Calculation of glutamate concentration}

The peak area of glutamate from the HPLC was integrated and used as an external standard for the samples. The glutamate concentration for $1 \mathrm{~g}$ of brain tissue was then calculated according to the sample quality.

\section{Determination of BBB permeability Experimental methods}

Evans blue was used as a tracer to measure BBB permeability. Two hours before each experiment, 2\% Evans blue $(2 \mathrm{ml} / \mathrm{kg})$ was injected into the ear vein. After $2 \mathrm{~h}$, the brain tissue was quickly removed. The tissue surrounding the hematoma was weighed (within $0.1 \mathrm{mg}$ ) and then placed into a test tube with $4 \mathrm{ml}$ formamide. The tube was then capped and placed in a $54^{\circ} \mathrm{C}$ water bath for 24 h to allow the Evans blue to spread throughout the brain tissue.

The samples were then centrifuged at 2,400 rpm for $5 \mathrm{~min}$. The supernatant was placed in a quartz cuvette, and a spectrophotometer was used $(\lambda=632 \mathrm{~nm})$ to measure the absorbance of the supernatant. Formamide was used as a blank control.

\section{Setting up the standard curve}

Evans blue ( $4 \mathrm{mg}$ ) was placed into a volumetric flask and weighed (within $0.1 \mathrm{mg}$ ). A total of $100 \mathrm{ml} \mathrm{NS}$ was added, and the solution was stirred. From this solution, $0.3 \mathrm{ml}$ was removed and placed in $5.7 \mathrm{ml}$ of formamide to make the standard buffer solution. A total of $3 \mathrm{ml}$ of this solution was serially diluted in seven tubes each containing $3 \mathrm{ml}$ formamide. The amount of Evans blue in each of the seven tubes was $8,4,2,1,0.5,0.25$ and $0.125 \mu \mathrm{g} / \mathrm{ml}$. The tubes were capped and placed into a $54^{\circ} \mathrm{C}$ water bath for 24 hours. The aforementioned method was used to measure absorbance. Linear regressions were calculated for the absorbencies and Evans blue content. The final equation was $y=0.0053 x+0.0608$ $(\mathrm{R} 2=0.9833)$.

\section{Evans blue content computation}

We used the formamide method to measure Evans blue content in brain tissue to assess BBB damage severity. The formula used was as follows: Evans blue content in brain tissue $(\mu \mathrm{g} / \mathrm{g}$ wet brain) $=\mathrm{B} \times$ formamide $(\mathrm{ml}) /$ wet weight $(\mathrm{g})$, where $\mathrm{B}$ refers to the Evans blue content of the sample $(\mu \mathrm{g} / \mathrm{ml})$ given by the linear regression equation according to standard curve.

\section{Measurement of BWC}

The dry and wet weight method was used to measure BWC. The brains were quickly removed, and brain tissue from the back-outside portion of the hematomas was used. First, the weight of the wet tissue was obtained. The samples were then placed in an oven at $100^{\circ} \mathrm{C}$ for $48 \mathrm{~h}$, and the dried samples were then weighed. BWC was then calculated as (wet weight-dry weight)/wet weight $\times 100 \%$.

\section{Statistical analysis}

All data were analyzed using SPSS 11.5. Basic data are expressed as the mean \pm standard deviation $(\mathrm{X} \pm \mathrm{SD})$. A Kruskal-Wallis test was used to make comparisons across the whole time series among the groups. If the Kruskal-Wallis test detected significant differences, Bonferroni corrections were used to make comparisons between groups. A correlative analysis was also performed to assess the relationship between the glutamate level and the $\mathrm{BBB}$ permeability. A $p$ value less than 0.05 was considered statistically significant. Statistical analysis was performed with the help of the Department of Biostatistics of Guiyang Medical College.

\section{Results}

Following blood infusion into the basal ganglia, the animals were unable to stand up or crawl. The contralateral extremities were less responsive to noxious stimulation. Brain CT showed an oval or round hyperdensity in the basal ganglia (Figure 1). The abovementioned observations demonstrate that the $\mathrm{ICH}$ model in this study was successful and reliable.

Three rabbits were excluded from this study, one animal died of overdose of anesthetic agents during surgery, and the other two did not show evidence of hematoma formation. A total of 33 rabbits in the ICH group (5 in the $6 \mathrm{~h}$ group, 6 in the $12 \mathrm{~h}$ group, 6 in the $18 \mathrm{~h}$ group, 5 in the $24 \mathrm{~h}$ group, 5 in the $48 \mathrm{~h}$ group and 6 in $72 \mathrm{~h}$ group) and 36 rabbits in the NC group were included in the present study.

\section{Hematoma volume and neurological function}

The hematoma volume and neurological deficit score in each subgroup were determined after the model was successfully induced. The average hematoma volume ranged from $0.250 \pm 0.014 \mathrm{ml}$ to $0.266 \pm 0.012 \mathrm{ml}$, and no significant difference was observed among subgroups. The neurological deficit score $(8.833 \pm 0.753$ to $9.167 \pm 0.817$ ) increased immediately after the blood was infused in the $\mathrm{ICH}$ group compared with the $\mathrm{NC}$ group, and no remarkable difference was noted among subgroups. These results suggested that the $\mathrm{ICH}$ model was successful. 

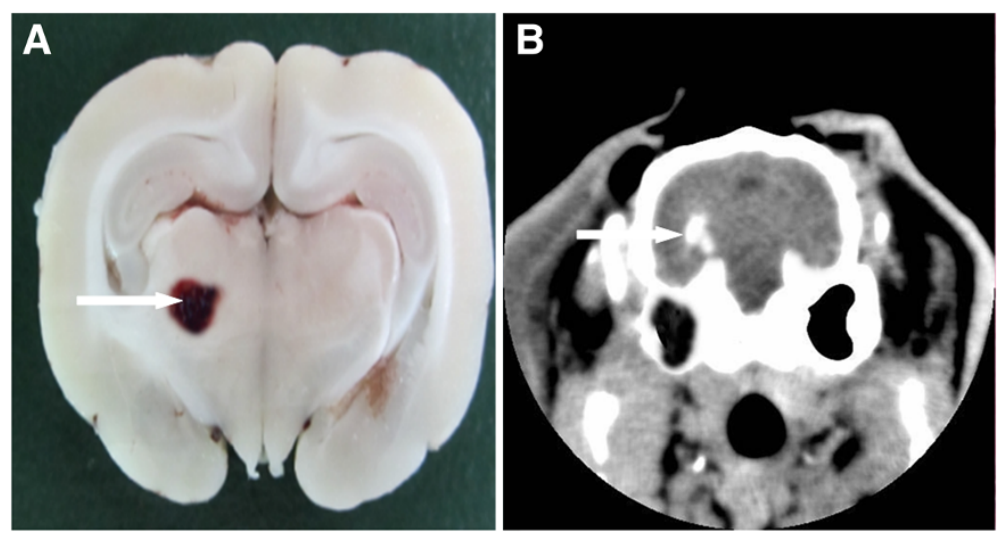

Figure 1 Brain CT and histological section showing hematoma in the rabbit's brain. The arrow pointed to the basal ganglia hematoma on histological section (A) or brain CT (B).

\section{Perihematomal glutamate level}

The glutamate level began to increase 6 hours after blood infusion. It gradually increased over time and peaked at 48 hours, which was significantly different compared with the NC group $(p<0.05)$. The glutamate level changed over time after blood was infused into the brain $\left(\chi^{2}=20.134\right)$. When comparisons were performed between subgroups, the glutamate level was lowest in 6hour group, also the Evans blue $\left(\chi^{2}=20.134\right)$ and the BWC $\left(\chi^{2}=20.142\right)$. These values were highest in $48-\mathrm{h}$ group (Wilcoxon $\mathrm{W}=27$ when the 6-h group was compared with the 48-h group, Wilcoxon $\mathrm{W}=21$ when the 6-h group was compared with the other groups), suggesting that less brain damage had occurred in the early stage after $\mathrm{ICH}$, indicating that it might be an optimal time window for intervention (Table 1).

In the NC group, no significant differences were observed between subgroups at different time points $(p>0.05)$, suggesting that the simple puncture of brain tissue with a needle has little impact on glutamate levels in brain tissue around the needle tract (Table 1).

\section{BBB permeability and BWC}

Evans blue and BWC around the hematoma began to increase 6 hours after successful ICH induction, increased gradually over time after blood infusion and peaked at 48 hours (Wilcoxon $\mathrm{W}=27$ when the $6 \mathrm{~h}$ group was compared with $48 \mathrm{~h}$ group, Wilcoxon $\mathrm{W}=21$ as $6 \mathrm{~h}$ group was compared with the other groups), suggesting that BBB disruption began in the early stage. Compared with the NC group, significant differences were observed for Evans blue content at different time points (at $6 \mathrm{~h}$, $12 \mathrm{~h}, 18 \mathrm{~h}, 24 \mathrm{~h}, 48 \mathrm{~h}$ and $72 \mathrm{~h}$ ) ,also the brain water content The same phenomenon was observed in perihematomal brain water content.

These results suggest that BBB permeability and brain edema both increased (Table 1). The range of the brain edema was observed on histological sections. Tissue approximately $5 \mathrm{~mm}$ around the hematoma became edematous, but the results were not quantified.

No significant difference was observed in Evans blue content or BWC among subgroups at different time points $(p>0.05)$ in the NC group, suggesting that a simple puncture of brain tissues does not decrease BBB permeability around the needle tract.

\section{Correlation between glutamate level and BBB disruption}

A correlation analysis was performed to analyze the relationship between perifocal glutamate levels and BBB permeability. Positive correlations was observed between

Table 1 Changes of perifocal glutamate level, Evan's blue and BEC in a rabbit model of ICH

\begin{tabular}{|c|c|c|c|c|c|c|}
\hline \multirow[t]{2}{*}{ Time point } & \multicolumn{2}{|c|}{ Glutamate content $(\mu \mathrm{g} / \mathrm{g})$} & \multicolumn{2}{|c|}{ Evan`s blue content $(\mu \mathrm{g} / \mathrm{g})$} & \multicolumn{2}{|c|}{ Brain water content(\%) } \\
\hline & $\mathrm{ICH}$ group & NC group & $\mathrm{ICH}$ group & NC group & $\mathrm{ICH}$ group & NC group \\
\hline 6 hour & $2286.34 \pm 43.78 \diamond \#$ & $2219.11 \pm 33.67$ & $28.86 \pm 0.87 \diamond \#$ & $4.14 \pm 0.21$ & $78.14 \pm 0.74 \diamond \#$ & $77.17 \pm 0.44$ \\
\hline 12 hour & $2317.64 \pm 65.18$ & $2184.51 \pm 47.12$ & $29.19 \pm 1.06$ & $3.96 \pm 0.13$ & $79.86 \pm 0.54$ & $77.06 \pm 0.43$ \\
\hline 18 hour & $2361.54 \pm 51.84$ & $2231.32 \pm 39.54$ & $32.19 \pm 0.74$ & $4.21 \pm 0.25$ & $81.19 \pm 0.41$ & $76.98 \pm 0.81$ \\
\hline 24 hour & $2438.62 \pm 42.56$ & $2213.98 \pm 50.38$ & $33.76 \pm 1.18$ & $4.17 \pm 0.18$ & $81.65 \pm 0.82$ & $77.45 \pm 0.91$ \\
\hline 48 hour & $2479.13 \pm 59.67$ & $2198.36 \pm 42.87$ & $35.36 \pm 1.18$ & $4.08 \pm 0.27$ & $81.92 \pm 0.68$ & $77.38 \pm 0.67$ \\
\hline 72 hour & $2480.23 \pm 49.60$ & $2210.88 \pm 45.66$ & $34.96 \pm 1.20$ & $4.20 \pm 0.22$ & $81.96 \pm 0.53$ & $77.43 \pm 0.78$ \\
\hline
\end{tabular}

$\diamond$ Compared with the NC group $(\mathrm{P}<0.05)$. \#Compared with other subgroups $(\mathrm{P}<0.05)$. 
elevated glutamate levels and BBB permeability $(r=0.948$, $p<0.05)$ and between glutamate level and BWC $(\mathrm{r}=0.841$, $p<0.05)$. The same correlation was also observed between $\mathrm{BBB}$ permeability and BWC $(\mathrm{r}=0.841, p<0.05)$. We found that the higher glutamate levels were associated with more serious BBB disruption and cerebral edema, suggesting that the perihematomal glutamate level was highly associated with BBB disruption and brain edema formation (Figure 2). Further observation demonstrated that the glutamate vs. Evans blue and glutamate vs. BWC plots display a sigmoidal relationship.

\section{Discussion}

Intracerebral hemorrhage remains the least treatable form of stroke (Rincon \& Mayer 2008). ICH models serve as an important tool for understanding mechanisms underlying brain injury after an intracerebral bleed (Andaluz et al. 2002). Based on previously published studies of an ICH model (Andaluz et al. 2002; Frantzias et al. 2011), we successfully generated a rabbit model of ICH by stereotactic injection of $0.3 \mathrm{ml}$ nonanticoagulant autologous arterial blood into the basal ganglia. The volume of the rabbit's brain is approximately 1 percent of the human brain (Narushima et al.
2003), so this injection is approximately equivalent to about $30 \mathrm{ml}$ of hematoma in the human brain. In the present study, an $\mathrm{ICH}$ model was induced successfully in 33 rabbits out of 36 ones. Three rabbits were excluded from the study as there is no evidence of hematoma formation. For experimental purposes, the most common technique of producing an intracerebral hematoma in rabbits is the injection of unclotted autologous blood. Sometimes, the injected blood may ruptures into the ventricular system or it extends to the subarachnoid or subdural space (Deinsberger et al. 1996). Therefore, a CT scan was performed after successful ICH injection 3 hours later. If postoperative brain CT does not show an oval or round hyperdensity in the basal ganglia,we do not consider that there is a hematoma formation.

$\mathrm{ICH}$ is always followed by blood-brain barrier disruption, which contributes to the enhanced BBB permeability and vasogenic brain edema in the surrounding areas. Brain edema is one of the most frequent and serious complications of $\mathrm{ICH}$, but how the $\mathrm{ICH}$ causes brain edema remains unknown (Wu et al. 2008). Previously published reports demonstrated that a range of factors are involved in BBB disruption (Keep et al. 2008). Studies in recent years have observed a relationship between

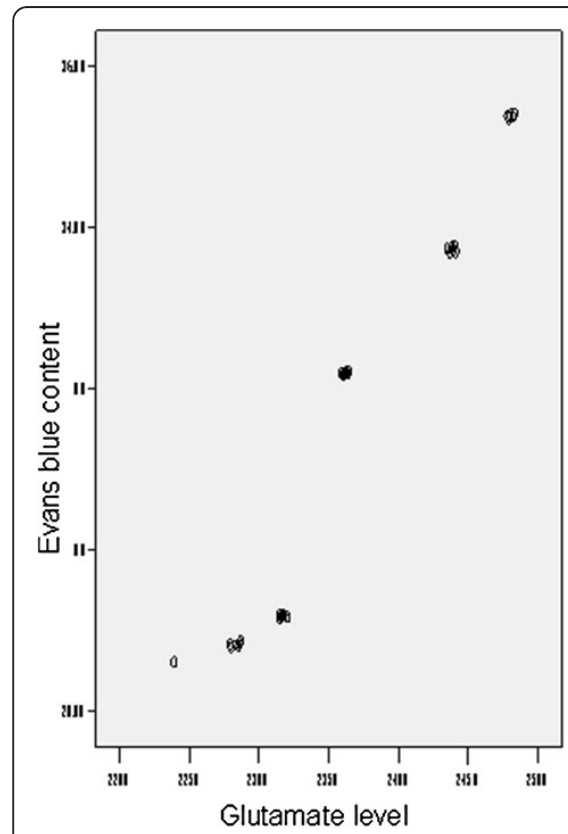

A:The correlation between the
glutamate and the Evans blue
content. $r=0.948, P<0.05$

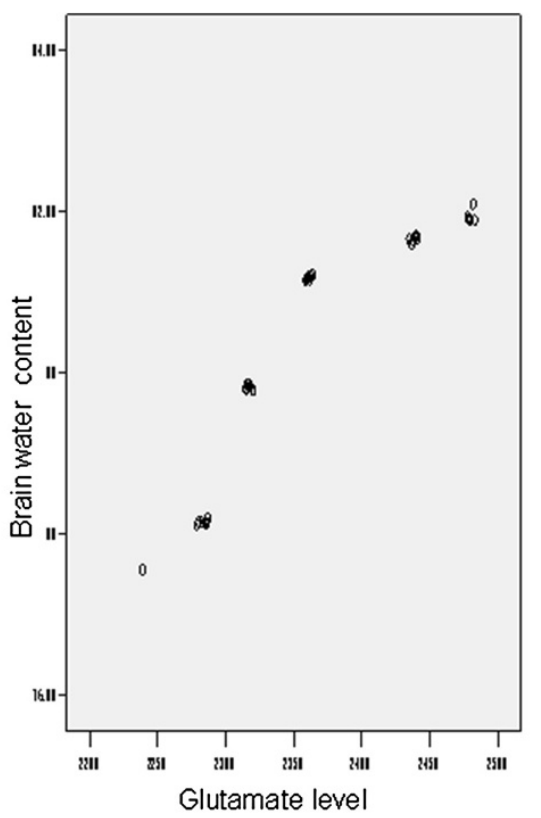

B:The correlation between the glutamate and the brain water content. $r=0.841, P<0.05$

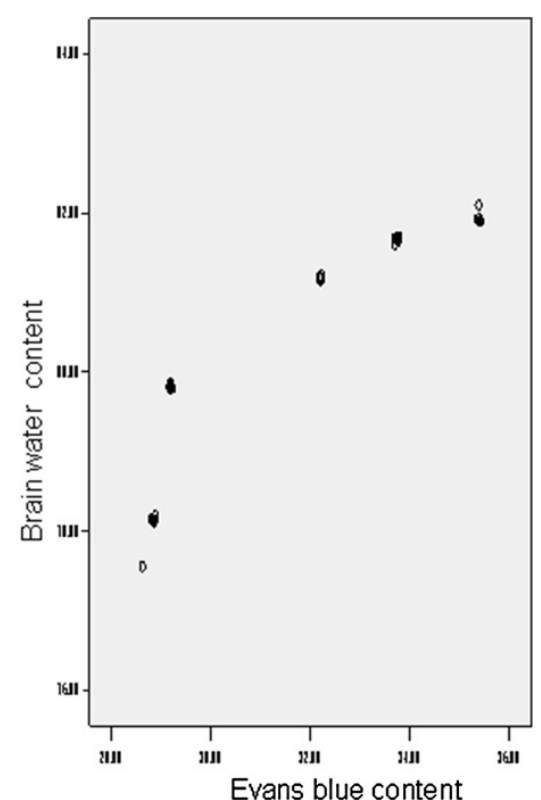

C:The correlation between the Evans blue and the brain water content. $r=0.843, P<0.05$

Figure 2 Correlation between the glutamate level and BBB disruption and brain edema formation. The perihematomal glutamate content has a positive correlation with the Evans blue content $(\mathbf{A})$ and the BWC (B). The same correlation was also observed between the the Evans blue content and the BWC (C). 
perihematomal brain tissue glutamate levels and secondary brain injury in models of $\mathrm{ICH}$ and the impact of glutamate on $\mathrm{ICH}$ patient outcome (Miller et al. 2007; Chiang et al. 2006; Hartings et al. 2008; Qureshi et al. 2003; Wang et al. 2008a). Glutamate is accumulated during the acute period of experimental hematoma, and the activation of NMDA receptors by glutamate can result in calcium influx and neuronal death following $\mathrm{ICH}$ (Lee et al. 2006). Secondary brain damages are associated with glutamate-related excitotoxicity, and the level of glutamate is closely associated with $\mathrm{ICH}$ patient outcome. Patients with spontaneous $\mathrm{ICH}$ who presented with more serious brain injuries had a higher concentration of glutamate in the brain and poorer prognosis (Wang et al. 2008b).

In the present study, perifocal glutamate levels began to increase 6 hours after infusing blood into the basal ganglia. As the time after infusing the blood into the brain was prolonged, the glutamate level increased gradually and peaked 48 hours after successful ICH induction. The perifocal glutamate level varied in subgroups at different time points. It was lowest in the 6 -h group and highest in the 48 and $72 \mathrm{~h}$ group. We observed statistically significant increases of perihematomal glutamate at different time points compared with the $\mathrm{NC}$ group, suggesting that glutamate level increase is a pathological process that follows $\mathrm{ICH}$.

Evans blue is a type of dark blue powdered dye that is unable to penetrate the BBB in normal conditions. The Evans blue assay is a popular method for quantifying BBB disruption. A small amount of Evans blue was observed in normal animal brains after intravenous injection (Wu et al. 2011). In the present studies, perihematomal Evans blue content was significantly increased in the ICH groups at different time points compared with the control group, demonstrating that the $\mathrm{BBB}$ was disrupted after the intracerebral infusion of blood. BBB disruption was followed by brain edema formation. Perihematomal BWC began to increase in the 6-h group and peaked 48 hours after ICH was induced. The change of BWC was consistent with enhanced $\mathrm{BBB}$ permeability. The range of edematous brain area was observed on histological sections. An area extending at least $5 \mathrm{~mm}$ from the hematoma became edematous, but because we only collected a total of $5 \mathrm{~mm}$ of brain tissue around the area to measure BWC, we were unable to precisely quantify the range of brain edema. This is a limitation of the present study.

The increased perifocal glutamate positively correlated with enhanced BBB permeability and increased BWC. Higher perihematomal glutamate levels were indicative of more serious BBB disruption and brain edema. Further observation demonstrated that the glutamate vs. Evans blue and glutamate vs. BWC plots display a sigmoidal relationship.suggesting that the $\mathrm{BBB}$ permeability or the
BWC increased slightly in the early stage as the glutamate level elevated. They increased dramatically as the glutamate level continue to increase. However, the increase of the BBB permeability or the WBC become lower after the glutamate level reaches to a certain extent. The relationship between the Evans blue and the BWC also display similar results.

However, the mechanism underlying increased perihematomal glutamate remains poorly understood. One possibility is an influx of glutamate from the initial hematoma into the extracellular space during ICH (Wagner et al. 1998). It is also possible that glutamate enters perihematomal brain tissue from the bloodstream following BBB disruption. Although the mechanism behind increased glutamate after $\mathrm{ICH}$ remains poorly understood, glutamate has been demonstrated to be involved in BBB disruption (Fu et al. 2007). It is reasonable for us to postulate that perihematomal $\mathrm{BBB}$ disruption is due to increased glutamate content. High levels of the glutamate activate NMDA receptors, consequently resulting in the entry of large amount of $\mathrm{Ca}^{2+}$ into the neurons, resulting in neuronal necrosis or apoptosis (Nicholls et al. 2007). Elevated glutamate levels in perihematomal brain tissues resulted in increase $\mathrm{BBB}$ disruption and permeability, thereby aggravating brain edema. Our results corroborate these findings. Because perihematomal glutamate level is closely associated with BBB disruption and brain edema formation, reducing glutamate levels or antagonizing its effect in the early stages of $\mathrm{ICH}$ might be effective in treating secondary brain edema. However, the current study focused on the relationship of glutamate levels and the $\mathrm{BBB}$ permeability, and because the animals were sacrificed 72 hours after ICH induction, we were unable to observe a correlation between glutamate levels and neurofunctional recovery.

\section{Competing interests}

The authors declare that they have no competing interests.

\section{Authors' contributions}

GW and SS designed the experiment and drafted the manuscript. FS and AF prepared the $\mathrm{ICH}$ model, and performed the statistic analyis. LW carried out the experiment to detect the glutmate, Evens blue and brain water content. All authors read and approved the final manuscript.

\section{Acknowledgements}

This research was partially supported by Guizhou Science and Technology Fund (2005-2048). We are indebted to the medical imaging department of Affiliated Hospital, Guiyang Medical College, for valuable discussions about the $\mathrm{ICH}$ model. We also wish to thank all the involved physicians for their hard work during the study.

\section{Author details}

${ }^{1}$ Department of Neurology, Affiliated Hospital, Guiyang Medical College, No. 28, Guiyijie Road, Liuguangmen, Guiyang City, Guizhou Province 550004, P R China. 'Emergency Department of Affiliated East Hospital, Tongji University, Shanghai 200120, P R China.

Received: 11 February 2013 Accepted: 8 July 2013

Published: 30 July 2013 


\section{References}

Andaluz N, Zuccarello M, Wagner KR (2002) Experimental animal models of intracerebral hemorrhage. Neurosurg Clin N Am 13:385-393

Chiang MF, Chiu WT, Lin FJ, Thajeb P, Huang CJ, Tsai SH (2006) Multiparametric analysis of cerebral substrates and nitric oxide delivery in cerebrospinal fluid in patients with intracerebral haemorrhage: correlation with hemodynamics and outcome. Acta Neurochir (Wien) 148:615-621, dicussion 621

Deinsberger W, Vogel J, Kuschinsky W, Auer LM, Boker DK (1996) Experimental intracerebral hemorrhage: description of a double injection model in rats. Neurol Res 18:475-477

Frantzias J, Sena ES, Macleod MR, Al-Shahi SR (2011) Treatment of intracerebral hemorrhage in animal models: meta-analysis. Ann Neurol 69:389-399

Fu H, Li W, Liu Y et al (2007) Mitochondrial proteomic analysis and characterization of the intracellular mechanisms of bis(7)-tacrine in protecting against glutamate-induced excitotoxicity in primary cultured neurons. J Proteome Res 6:2435-2446

Germano A, Caffo M, Angileri FF et al (2007) NMDA receptor antagonist felbamate reduces behavioral deficits and blood-brain barrier permeability changes after experimental subarachnoid hemorrhage in the rat. J Neurotrauma 24:732-744

Hartings JA, Gugliotta M, Gilman C, Strong AJ, Tortella FC, Bullock MR (2008) Repetitive cortical spreading depolarizations in a case of severe brain trauma. Neurol Res 30:876-882

Hazell AS (2007) Excitotoxic mechanisms in stroke: an update of concepts and treatment strategies. Neurochem Int 50:941-953

Imer M, Omay B, Uzunkol A et al (2009) Effect of magnesium, MK-801 and combination of magnesium and MK-801 on blood-brain barrier permeability and brain edema after experimental traumatic diffuse brain injury. Neurol Res 31:977-981

Keep RF, Xiang J, Ennis SR et al (2008) Blood-brain barrier function in intracerebral hemorrhage. Acta Neurochir Suppl 105:73-77

Lampl Y, Shmuilovich O, Lockman J, Sadeh M, Lorberboym M (2005) Prognostic significance of blood brain barrier permeability in acute hemorrhagic stroke Cerebrovasc Dis 20:433-437

Lee ST, Chu K, Jung KH et al (2006) Memantine reduces hematoma expansion in experimental intracerebral hemorrhage, resulting in functional improvement. J Cereb Blood Flow Metab 26:536-544

Miller CM, Vespa PM, McArthur DL, Hirt D, Etchepare M (2007) Frameless stereotactic aspiration and thrombolysis of deep intracerebral hemorrhage is associated with reduced levels of extracellular cerebral glutamate and unchanged lactate pyruvate ratios. Neurocrit Care 6:22-29

Narushima I, Kita T, Kubo K et al (2003) Highly enhanced permeability of bloodbrain barrier induced by repeated administration of endothelin-1 in dogs and rats. Pharmacol Toxicol 92:21-26

Nicholls DG, Johnson-Cadwell L, Vesce S, Jekabsons M, Yadava N (2007) Bioenergetics of mitochondria in cultured neurons and their role in glutamate excitotoxicity. J Neurosci Res 85:3206-3212

Parker D Jr, Rhoney DH, Liu-DeRyke X (2010) Management of spontaneous nontraumatic intracranial hemorrhage. J Pharm Pract 23:398-407

Purdy PD, Devous MD Sr, Batjer HH, White CL 3rd, Meyer Y, Samson DS (1989) Microfibrillar collagen model of canine cerebral infarction. Stroke 20:1361-1367

Qureshi Al, Ali Z, Suri MF et al (2003) Extracellular glutamate and other amino acids in experimental intracerebral hemorrhage: an in vivo microdialysis study. Crit Care Med 31:1482-1489

Rincon F, Mayer SA (2008) Current treatment options for intracerebral hemorrhage. Curr Treat Options Cardiovasc Med 10:229-240

Shi W, Wang Z, Pu J et al (2011) Changes of blood-brain barrier permeability following intracerebral hemorrhage and the therapeutic effect of minocycline in rats. Acta Neurochir Suppl 110:61-67

Wagner KR, Xi G, Hua Y, Kleinholz M, de Courten-Myers GM, Myers RE (1998) Early metabolic alterations in edematous perihematomal brain regions following experimental intracerebral hemorrhage. J Neurosurg 88:1058-1065

Wang Z, Gardiner NJ, Fernyhough P (2008a) Blockade of hexokinase activity and binding to mitochondria inhibits neurite outgrowth in cultured adult rat sensory neurons. Neurosci Lett 434:6-11

Wang E, Ho CL, Lee KK, Ng I, Ang BT (2008b) Effects of temperature changes on cerebral biochemistry in spontaneous intracerebral hematoma. Acta Neurochir Suppl 102:335-338
Wu H, Zhao R, Qi J et al (2008) The expression and the role of protease nexin-1 on brain edema after intracerebral hemorrhage. J Neurol Sci 270:172-183

Wu G, Li C, Wang L, Mao Y, Hong Z (2011) Minimally invasive procedures for evacuation of intracerebral hemorrhage reduces perihematomal glutamate content, blood-brain barrier permeability and brain edema in rabbits. Neurocrit Care 14:118-126

doi:10.1186/2193-1801-2-358

Cite this article as: Wu et al:: Perihematomal glutamate level is associated with the blood-brain barrier disruption in a rabbit model of intracerebral hemorrhage. SpringerPlus 2013 2:358.

\section{Submit your manuscript to a SpringerOpen ${ }^{\circ}$ journal and benefit from:}

- Convenient online submission

- Rigorous peer review

- Immediate publication on acceptance

- Open access: articles freely available online

High visibility within the field

- Retaining the copyright to your article

Submit your next manuscript at $\gg$ springeropen.com 